\title{
Comparative analysis of mitochondrial genomes between a wheat K-type cytoplasmic male sterility (CMS) line and its maintainer line
}

Huitao Liu' ${ }^{1,5}$, Peng Cui ${ }^{2+}$, Kehui Zhan ${ }^{3}$, Qiang Lin ${ }^{2,5}$, Guoyin Zhuo ${ }^{1,4}$, Xiaoli Guo ${ }^{4}$, Feng Ding ${ }^{2}$, Wenlong Yang ${ }^{1}$ Dongcheng Liu', Songnian $\mathrm{Hu}^{2}$, Jun $\mathrm{Yu}^{2^{*}}$ and Aimin Zhang ${ }^{1^{*}}$

\begin{abstract}
Background: Plant mitochondria, semiautonomous organelles that function as manufacturers of cellular ATP, have their own genome that has a slow rate of evolution and rapid rearrangement. Cytoplasmic male sterility (CMS), a common phenotype in higher plants, is closely associated with rearrangements in mitochondrial DNA (mtDNA), and is widely used to produce F1 hybrid seeds in a variety of valuable crop species. Novel chimeric genes deduced from mtDNA rearrangements causing CMS have been identified in several plants, such as rice, sunflower, pepper, and rapeseed, but there are very few reports about mtDNA rearrangements in wheat. In the present work, we describe the mitochondrial genome of a wheat K-type CMS line and compare it with its maintainer line.

Results: The complete mtDNA sequence of a wheat K-type (with cytoplasm of Aegilops kotschyi) CMS line, Ks3, was assembled into a master circle (MC) molecule of 647,559 bp and found to harbor 34 known protein-coding genes, three rRNAs (18 S, $26 \mathrm{~S}$, and $5 \mathrm{~S}$ rRNAs), and 16 different tRNAs. Compared to our previously published sequence of a K-type maintainer line, Km3, we detected Ks3-specific mtDNA (> 100 bp, 11.38\%) and repeats (> 100 bp, 29 units) as well as genes that are unique to each line: $r p / 5$ was missing in $\mathrm{Ks} 3$ and $\mathrm{trnH}$ was absent from $\mathrm{Km} 3$. We also defined 32 single nucleotide polymorphisms (SNPs) in 13 protein-coding, albeit functionally irrelevant, genes, and predicted 22 unique ORFs in Ks3, representing potential candidates for K-type CMS. All these sequence variations are candidates for involvement in CMS. A comparative analysis of the mtDNA of several angiosperms, including those from Ks3, Km3, rice, maize, Arabidopsis thaliana, and rapeseed, showed that non-coding sequences of higher plants had mostly divergent multiple reorganizations during the mtDNA evolution of higher plants.

Conclusion: The complete mitochondrial genome of the wheat K-type CMS line Ks3 is very different from that of its maintainer line $\mathrm{Km} 3$, especially in non-coding sequences. Sequence rearrangement has produced novel chimeric ORFs, which may be candidate genes for CMS. Comparative analysis of several angiosperm mtDNAs indicated that non-coding sequences are the most frequently reorganized during mtDNA evolution in higher plants.
\end{abstract}

\section{Background}

Mitochondria, as semiautonomous organelles, function as manufacturers of cellular ATP through the process of oxidative phosphorylation in all eukaryotes. It is believed that mitochondria originated from a free-living eubacterial

\footnotetext{
* Correspondence: junyu@big.ac.cn; amzhang@genetics.ac.cn

+ Contributed equally

'The State Key Laboratory of Plant Cell and Chromosome Engineering, Institute of Genetics and Developmental Biology, Chinese Academy of Sciences, Beijing, 100101, China

${ }^{2}$ The CAS Key Laboratory of Genome Sciences and Information, Beijing Institute of Genomics, Chinese Academy of Sciences, Beijing, 100029, China Full list of author information is available at the end of the article
}

ancestor and became an endosymbiotic organelle through engulfment by a eukaryotic host cell [1,2]. The sizes of mitochondrial genomes (mtDNA) vary among eukaryotes, ranging from $6 \mathrm{~kb}$ in Plasmodium to $200-2000 \mathrm{~kb}$ in higher plants $[3,4]$. Due to frequent mtDNA recombination and extraneous DNA incorporation from the chloroplast (cp) and nuclear genomes, extensive size expansion of mtDNA in higher plants occurs very frequently. In higher plants, in addition to their large genome sizes, mtDNAs display distinctive features, including slow evolutionary rate, rapid rearrangement, frequent insertion, complex multipartite structure, specific mode of gene

\section{Biomed Central}


expression, cis-/trans-splicing, RNA editing, and use of the universal genetic code [5]. In higher plants, protein-coding genes in mtDNA are extremely conserved but their gene order and non-protein-coding sequences are rather variable [6-8], and their structural organization is very dynamic [9]. The dynamic multipartite structures in higher plants exhibit redundancy and copy number variation [10]. Gene shuffling and variations may result in different phenotypes, such as cytoplasmic male sterility (CMS) [11].

CMS is a common phenotype in higher plants, and is closely associated with mutations in mtDNAs that cause pollen abortion. CMS systems have been widely used as a convenient way to produce F1 hybrid seeds in a variety of valuable crop species, including rice, maize, sugar beet, and cotton. In addition, CMS is exploited to study nucleocytoplasmic interactions [12]. mtDNAs in higher plants are known to have the ability to undergo extensive recombination, resulting in sequence rearrangements. When these rearrangements produce "chimeric genes", they may directly or indirectly alter normal physiological functions, such as pollen abortion. Therefore, comparative analysis of mtDNAs between a CMS line and its normal fertile counterpart should lead to the molecular details underlying the sterility phenotype in higher plants.

Wheat K-type CMS, which lacks adverse cytoplasmic effects and has more restoration line resources than other types of CMS, has been widely used in the production of hybrid seeds. Moreover, we recently sequenced the complete mtDNA genome for fertile Yumai 3 (Triticum aestivum cv. Yumai 3, Km3), which is a maintainer line of K-type CMS [13]. In this study, we acquired and analyzed another complete mtDNA from a wheat K-type CMS line, Ks3, with the sterilizing cytoplasm derived from Aegilops kotschyi, Boiss.

\section{Results}

\section{Organization of $\mathrm{Ks} 3 \mathrm{mtDNA}$}

We acquired the Ks3 mtDNA sequence by exploiting a BAC-based cloning strategy, which yielded a circular molecule 647,559 bp in length with $44.3 \% \mathrm{G}+\mathrm{C}$ content (Figure 1). In this master circle (MC) molecule, there were four large repeat sequences of more than $20 \mathrm{~kb}$. The largest was 98,977 bp, extending from 63707 to 162682, including 22 genes (Figure 1 and Additional File 1). The actual Ks3 mtDNA was $400 \mathrm{~kb}$ as estimated by removing one copy each of the large repeats with more than 500 bp from the MC molecule. We used similarity searches (BLAST and tRNA scan-SE) and found 53 genes in total; among them, we identified 34 known protein-coding genes, three rRNAs $(18 \mathrm{~S}, 26 \mathrm{~S}$, and $5 \mathrm{~S}$ rRNAs), and 16 tRNAs, accounting for $6.22 \%$ of the genome (Additional File 1). In addition, using sequence

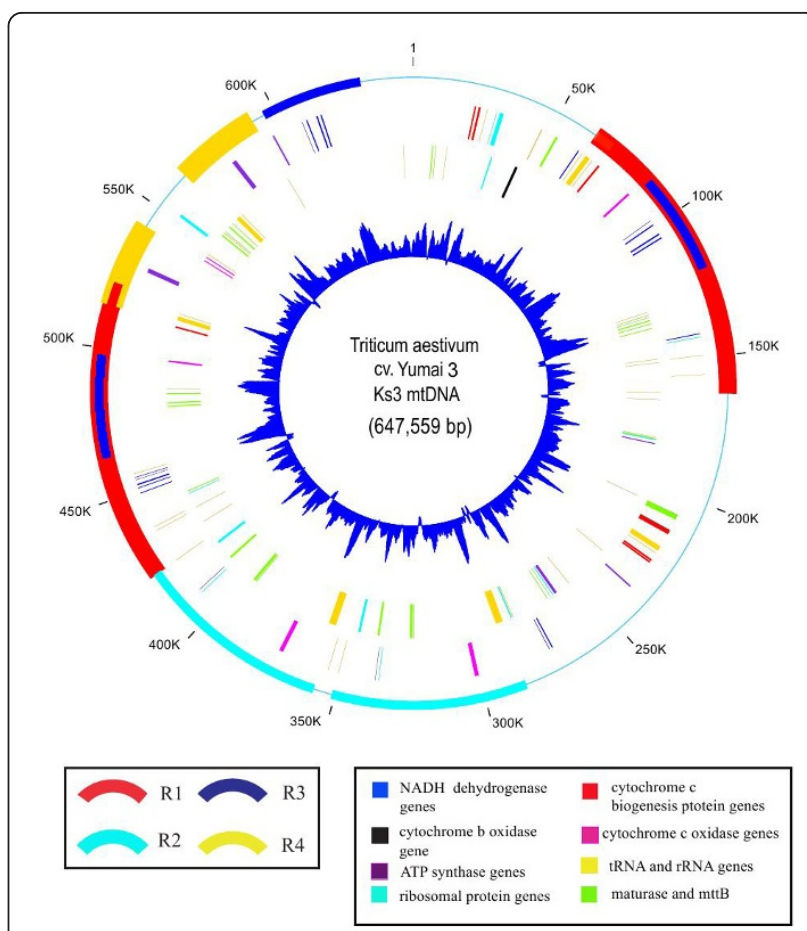

Figure 1 Physical map of the Triticum aestivum cv. Yumai $3 \mathrm{~K}$ type CMS line (Ks3) mitochondrial genome. Circles display (from outside): (1) physical map scaled in kilobase pairs; different colored arcs indicate repeats of more than $20 \mathrm{~kb}$, as in the legend shown in the bottom left corner: red, R1; blue-green, R2; blue, R3; golden yellow, R4; (2) and (3) coding sequences transcribed clockwise and counterclockwise, respectively: different colors represent the different genes, as in the legend shown in the bottom right corner: blue violet, ATP synthases; blue, NADH dehydrogenases; red, cytochrome c biogenesis proteins; purple-red, cytochrome c oxidases; black, cytochrome b oxidase; blue-green, ribosomal proteins; golden yellow, tRNA and rRNA genes; yellow-green, maturase and mttB; (4) GC content variations (in a 1000-bp window and $100-b p$ increments).

analysis, we classified 248 ORFs longer than 300 bp, which summed to $19.8 \%$ or 128,277 bp in total.

We also analyzed the transposable elements in Ks3 mtDNA using TIGR's transposable element database as a reference http://www.tigr.org/tdb/e2k1/plant.repeats/index. shtml with a minimum match of $50 \mathrm{bp}$. The results showed that there were 12 small fragments, ranging from $59 \mathrm{bp}$ to $230 \mathrm{bp}$, that were identical to known retrotransposons (Additional File 2). Ten retrotransposons were identical to those of rice and the remaining retrotransposons were identical to those of wheat, with identities ranging from $79 \%$ to $98 \%$. The overall length of the retrotransposons was $1476 \mathrm{bp}, 0.23 \%$ of the total Ks3 mtDNA.

\section{Ks3-specific mtDNA regions}

We compared Ks3 mtDNA with that of $\mathrm{Km} 3$ using BLAST2, and the analysis revealed 385,765 shared base 
pair, i.e., $85.2 \%$ of the total $\mathrm{Km} 3$ sequence. In addition, Ks3 mtDNA had a 574,215-bp sequence that was homologous to $\mathrm{Km} 3 \mathrm{mtDNA}$, accounting for $88.7 \%$ of the total. The conserved sequences in $\mathrm{Km} 3$ and $\mathrm{Ks} 3$ were broken into 43 and 44 sequence segments, respectively. We also revealed 38 segments (designated U1-U38) of more than 100 bp in Ks3 mtDNA that were not maintained in $\mathrm{Km} 3$ mtDNA (Figure 2 Additional Files 3 and 4) and totaled $73,670 \mathrm{bp}(11.38 \%)$, ranging in size from 120 to $6371 \mathrm{bp}$ and interspersed over 62 locations in the $\mathrm{Ks} 3 \mathrm{MC}$ molecule. It is notable that there were multiple copies in some specific regions. For example, there were four copies of U18, and three copies each of unique regions of $\mathrm{U} 1, \mathrm{U} 5$, and $\mathrm{U} 21$. Other unique regions had double or single copies. In the following description, the sum of the length of different specific regions includes every copy unless stated otherwise.

We annotated these $38 \mathrm{Ks} 3$-specific sequences using BlastN and BlastX searching against NCBI databases. Four integrated segments, U17, U18, U19, and U28, were found in the databases with a total of $6727 \mathrm{bp}$, while 10 segments $(10,445 \mathrm{bp})$ could not be detected, and 24 segments were partially annotated in 37 pieces $(19,590 \mathrm{bp})$. As a result, 26,317 bp were explained,

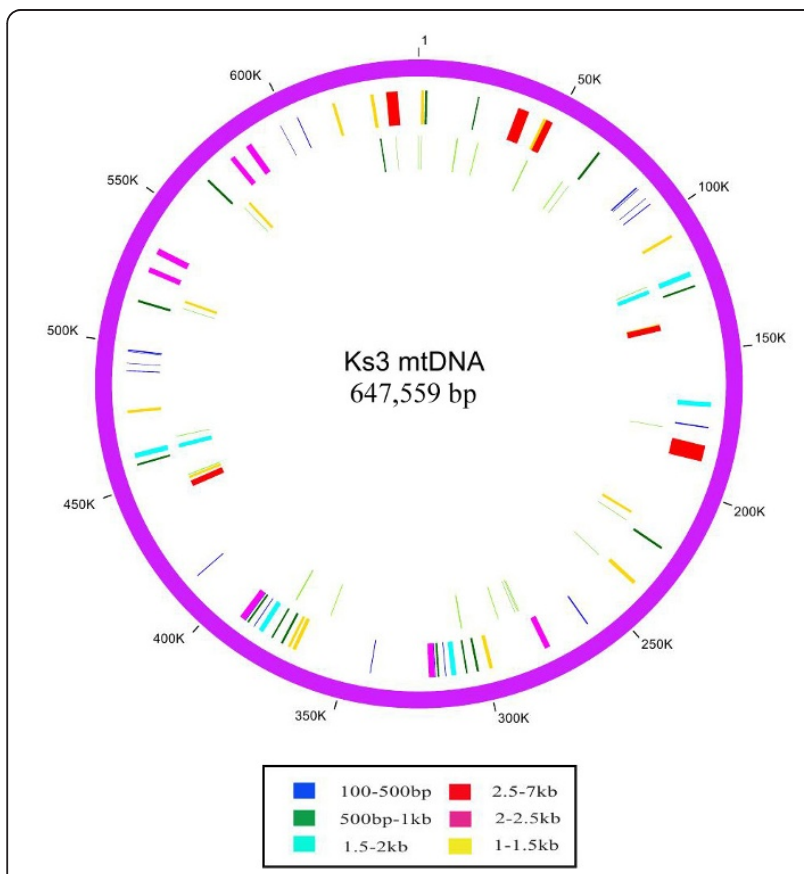

Figure 2 Distribution of unique sequences and sequences showing homology to wheat ctDNA sequences in Ks3 mtDNA. Circles display (from outside): (1) physical map scaled in kilobase pairs; (2) the locations of unique sequences; (3) the locations of chloroplast homologous sequences. Different colors represent different sequence lengths: blue, 100-500 bp; dark green, 500-1000 bp; golden yellow, 1-1.5 kb; blue-green, 1.5-2 kb; purple-red, 2-2.5 $\mathrm{kb}$; and red, 2.5-7 kb. accounting for $35.7 \%$ of Ks3-specific sequences and $4 \%$ of the Ks3 mitochondrial genome. Furthermore, $21 \mathrm{Ks} 3-$ specific segments (20,858 bp), ranging from 33 to 3301 $\mathrm{bp}$, were homologous to several previously determined mitochondrial sequences in higher plants, e.g., Zea mays, Sorghum bicolor, Oryza sativa, Bambusa oldhamii, and Tripsacum dactyloides. In addition, partial segments (3991 bp) in U23, U26, and U30 were found to be significantly homologous to wheat chloroplast DNA. Five segments, U8, U11, U14, U24, and U32, partially matched several nuclear genome sequences of different higher plants. Nevertheless, Ks3-specific regions, which accounted for about $47,353 \mathrm{bp}$ and $7.3 \%$ of $\mathrm{Ks} 3$ mtDNA, were novel to the current NCBI databases.

\section{Homology of Ks3 mtDNA to wheat ctDNA}

We analyzed homology between Ks3 mtDNA and the wheat chloroplast genome (ctDNA) using BLAST2, and revealed 123 segments $(25,714 \mathrm{bp}, 4 \%)$ with more than $81 \%$ identity (Additional File 5 ) and a size range of 24 to $2790 \mathrm{bp}$. Thirty-eight of these segments were more than $100 \mathrm{bp}$ in length, and summed to $21,040 \mathrm{bp}(3.2 \%)$ (Figure 2 Additional File 6).

We noticed that some segments in Ks3 mtDNA were homologous to wheat ctDNA with multiple copies; $\mathrm{Ct} 7$, $\mathrm{Ct11}$, Ct16, and $\mathrm{Ct} 17$ were duplicated and $\mathrm{Ct} 3$ has four copies. Fifty-six segments covered the full length or parts of known genes; six segments contained tRNA genes derived from ctDNA $(\operatorname{trn} S, \operatorname{trn} W, \operatorname{trn} C, \operatorname{trn} N-1$, $\operatorname{trn} N-2$, and $\operatorname{trn} N-3)$. The other 50 fragments were classified into $10 \mathrm{mtDNA}$-derived genes (3792 bp, 0.6\%): atp1, rrn18-1, rrn18-2, rrn18-3, rrn18-4, rrn26-1, rrn262 , $\operatorname{trn} M-1$, and $\operatorname{trn} M-2$, corresponding to wheat ctDNA genes atpA, rrn16, rrn23, and ct-trnM.

In addition, by comparing the wheat ctDNA homologies to Ks3 mtDNA with wheat ctDNA homologies to $\mathrm{Km} 3 \mathrm{mtDNA}$, we observed that most of these homologies with $\mathrm{Ks} 3$ and $\mathrm{Km} 3 \mathrm{mtDNA}$ were identical. Only two homologous segments (1930 bp) between wheat ctDNA and $\mathrm{Km} 3 \mathrm{mtDNA}$ were not shared with $\mathrm{Ks} 3$ mtDNA. Similarly, four segments (3991 bp) in Ks3 mtDNA were uniquely homologous with wheat ctDNA, and were located in Ks3-specific mtDNA regions, U23, U26, and U30 (Additional File 7). Additional File 6 also indicates that these unique homologous segments of Ks3 mtDNA and wheat ctDNA were contained in Ct18, Ct21, Ct24, and Ct24R. The results reveal that the mitochondrial genomes of $\mathrm{Ks} 3$ and $\mathrm{Km} 3$ may incorporate some specific extraneous DNA from the wheat chloroplast genome.

\section{Ks3 mtDNA repeat sequences}

The mtDNAs of higher plants harbor massive repeated sequences. In the Ks3 mtDNA, we defined 29 repeats 
(> $100 \mathrm{bp}$ ), comprising both direct (DR) and inverted repeats (IR) (Table 1); among them, nine involved two copies, twelve had three copies, and six had four copies. There were four large repeats, R1, R2, R3, and R4, which exceeded $20 \mathrm{~kb}$, with lengths of 98,977, 64,991, 33605 , and $28,476 \mathrm{bp}$, respectively. Other repeats were smaller in size and had distinct distributions and copy number variations (Figure 3 Additional File 8).

Plant mtDNA is known to contain multipartite structures [14-17]. The isomeric forms of the MC molecule and subgenomic circles are decipherable based on assumptions of intra-molecular homologous recombination [18].
We produced various molecular forms of the Ks3 MC molecule by intra-molecular recombination between different repeat pairs, including three DR of more than $10 \mathrm{~kb}$ and four IR of more than 8-kb (Figure 4). Other repeat pairs may also produce possible sites for additional recombination. These subgenomic structures are real. For tobacco mtDNA, subgenomic circles were directly observed using electron microscopy [19], and Sugiyama et al. [15] proved that long-range PCR could be used to test recombinant molecules formed by inter-molecule recombination.

Moreover, we also compared repeats between Ks3 mtDNA and $\mathrm{Km} 3 \mathrm{mtDNA}$. It is known that the $\mathrm{Km} 3$

Table 1 Repeats (> $100 \mathrm{bp}$ ) found in Ks3 mtDNA

\begin{tabular}{|c|c|c|c|c|c|c|c|c|}
\hline No. & Type $^{a}$ & Size (bp) & $\begin{array}{l}\text { MC coordinates } \\
\text { Copy-1 }\end{array}$ & Copy-2 & Copy-3 & Copy-4 & $\begin{array}{l}\text { Difference } \\
\text { between copies }\end{array}$ & {$\left[\% I^{2}\right]^{\mathrm{e}}$} \\
\hline R1 & $\mathbb{R}$ & 98977 & $63707-162682$ & $520734-421758$ & & & copy-1 1 bp del. ${ }^{c} ; 3$ bp mismatch ${ }^{d}$ & 99.99 \\
\hline $\mathrm{R} 2$ & DR & 64991 & $285968-350958$ & $357152-422142$ & & & identical & 100 \\
\hline R3 & $\mathrm{IR} / \mathrm{DR}$ & 33605 & $596378-629979$ & $497569-463965$ & $86872-120476$ & & copy-1 4 bp del.; 20 bp mismatch & 99.93 \\
\hline R4 & $\mathrm{DR}$ & 28476 & $563639-592113$ & $514143-542618$ & & & copy-1 1 bp del.; 3 bp mismatch & 99.99 \\
\hline R5 & IR & 8853 & $217390-226242$ & $521779-512927$ & & & 2 bp mismatch & 99.98 \\
\hline R6 & $\mathrm{DR} / \mathrm{IR}$ & 7808 & $218435-226242$ & $63707-71514$ & $520734-512927$ & & 3 bp mismatch & 99.96 \\
\hline R7 & $\mathrm{IR} / \mathrm{DR}$ & 7637 & $563639-571274$ & $225026-217390$ & $514143-521779$ & & copy-1 1 bp del.; 3 bp mismatch & 99.97 \\
\hline R8 & $\mathrm{IR} / \mathrm{DR}$ & 6592 & $563639-570299$ & $70298-63707$ & $514143-520734$ & $225026-218435$ & copy-1 1 bp del.; 4 bp mismatch & 99.92 \\
\hline R9 & DR & 4645 & 233766-238410 & 592108-596752 & & & $3 \mathrm{bp}$ mismatch & 99.94 \\
\hline $\mathrm{R} 10$ & $\mathrm{DR}$ & 442 & $238110-238550$ & 389430-389871 & $318246-318687$ & & copy-1 1 bp del.; 7 bp mismatch & 98.19 \\
\hline $\mathrm{R} 11$ & $\mathbb{R}$ & 409 & $178030-178438$ & $532798-532390$ & $582293-581885$ & & identical & 100 \\
\hline $\mathrm{R} 12$ & $\mathbb{R}$ & 385 & $162298-162682$ & $350958-350574$ & $422142-421758$ & & identical & 100 \\
\hline R13 & $\mathrm{DR} / \mathrm{IR}$ & 391 & $15949-16326$ & $570623-571013$ & $218041-217651$ & $521128-521518$ & copy-1 13 bp del.; 26 bp mismatch & 90.03 \\
\hline R14 & $\mathrm{IR} / \mathrm{DR}$ & 378 & $238036-238410$ & $497569-497192$ & $86872-87249$ & $596378-596752$ & copy-1 4 bp del.; 20 bp mismatch & 93.4 \\
\hline \multirow[t]{3}{*}{ R15 } & $\mathrm{DR} / \mathrm{IR}$ & 352 & $86898-87249$ & $318195-318546$ & 389379-389730 & $497543-497192$ & copy-1 1 bp del.; copy-5 4 bp del.; & 93.1 \\
\hline & & & $238062-238410$ & $596404-596752$ & & & 20 bp mismatch & \\
\hline & & & R15 Copy-5 & R15 Copy-6 & & & & \\
\hline R16 & $\mathrm{IR} / \mathrm{DR}$ & 295 & $42766-43060$ & $126790-126496$ & $457651-457945$ & & identical & 100 \\
\hline \multirow[t]{3}{*}{$\mathrm{R} 17$} & $\mathrm{DR} / \mathrm{IR}$ & 275 & $67932-68206$ & $114585-114859$ & $624088-624362$ & $469856-469582$ & identical & 100 \\
\hline & & & $222660-222934$ & $566004-565730$ & $516509-516235$ & & & \\
\hline & & & R17 Copy-5 & R17 Copy-6 & R17 Copy-7 & & & \\
\hline $\mathrm{R} 18$ & $\mathbb{R}$ & 246 & $2411-2646$ & $416690-416445$ & $345506-345261$ & & copy-1 10 bp del.; 5 bp mismatch & 94.31 \\
\hline R19 & $\mathrm{DR} / \mathrm{IR}$ & 201 & $546169-546367$ & $435151-435351$ & $149290-149090$ & & copy-1 2 bp del.; 2 bp mismatch & 98.01 \\
\hline $\mathrm{R} 20$ & $\mathrm{DR}$ & 197 & $182981-183177$ & $543334-543530$ & & & 1 bp mismatch & 99.49 \\
\hline $\mathrm{R} 21$ & $\mathrm{IR} / \mathrm{DR}$ & 193 & $265694-265885$ & $610987-610795$ & $482957-483149$ & 101484-101292 & copy-1 1 bp del.; 1 bp mismatch & 98.96 \\
\hline $\mathrm{R} 22$ & $\mathbb{R}$ & 190 & 13994-14183 & $63634-63445$ & & & 4 bp mismatch & 97.89 \\
\hline R23 & $\mathbb{R}$ & 189 & 271802-271990 & $420288-420100$ & $349104-348916$ & & identical & 100 \\
\hline R24 & $\mathrm{DR}$ & 185 & $13588-13772$ & $358007-358191$ & $286823-287007$ & & identical & 100 \\
\hline $\mathrm{R} 25$ & $\mathrm{DR} / \mathrm{IR}$ & 179 & 301013-301190 & $643373-643195$ & $372197-372374$ & & copy-1,3 1 bp del.; 9 bp mismatch & 94.41 \\
\hline $\mathrm{R} 26$ & $\mathrm{DR}$ & 154 & $260325-260478$ & $642452-642605$ & & & 4 bp mismatch & 97.4 \\
\hline R27 & $\mathrm{DR} / \mathrm{IR}$ & 154 & $91917-92070$ & $170372-170525$ & $492524-492371$ & $601420-601573$ & 8 bp mismatch & 94.81 \\
\hline $\mathrm{R} 28$ & DR & 145 & $37571-37703$ & $543007-543151$ & & & copy-1 12 bp del.; 2 bp mismatch & 90.34 \\
\hline R29 & $\mathrm{IR} / \mathrm{DR}$ & 119 & $529005-529122$ & $349116-348998$ & $420300-420182$ & $578500-578617$ & copy-1,4 1 bp del.; 2 bp mismatch & 97.48 \\
\hline
\end{tabular}

${ }^{\mathrm{a} D R}$ and IR: direct and reverse repeats, respectively; IR/DR: both direct repeat and reverse repeat among multiple copies.

${ }^{\mathrm{b}}$ Boldface: IR copy, compared with copy-1 as control.

cNumber of deleted (del.) bases compared to other copies.

${ }^{\mathrm{d}}$ Number of bases without match among the copies.

eIDY: identity. 


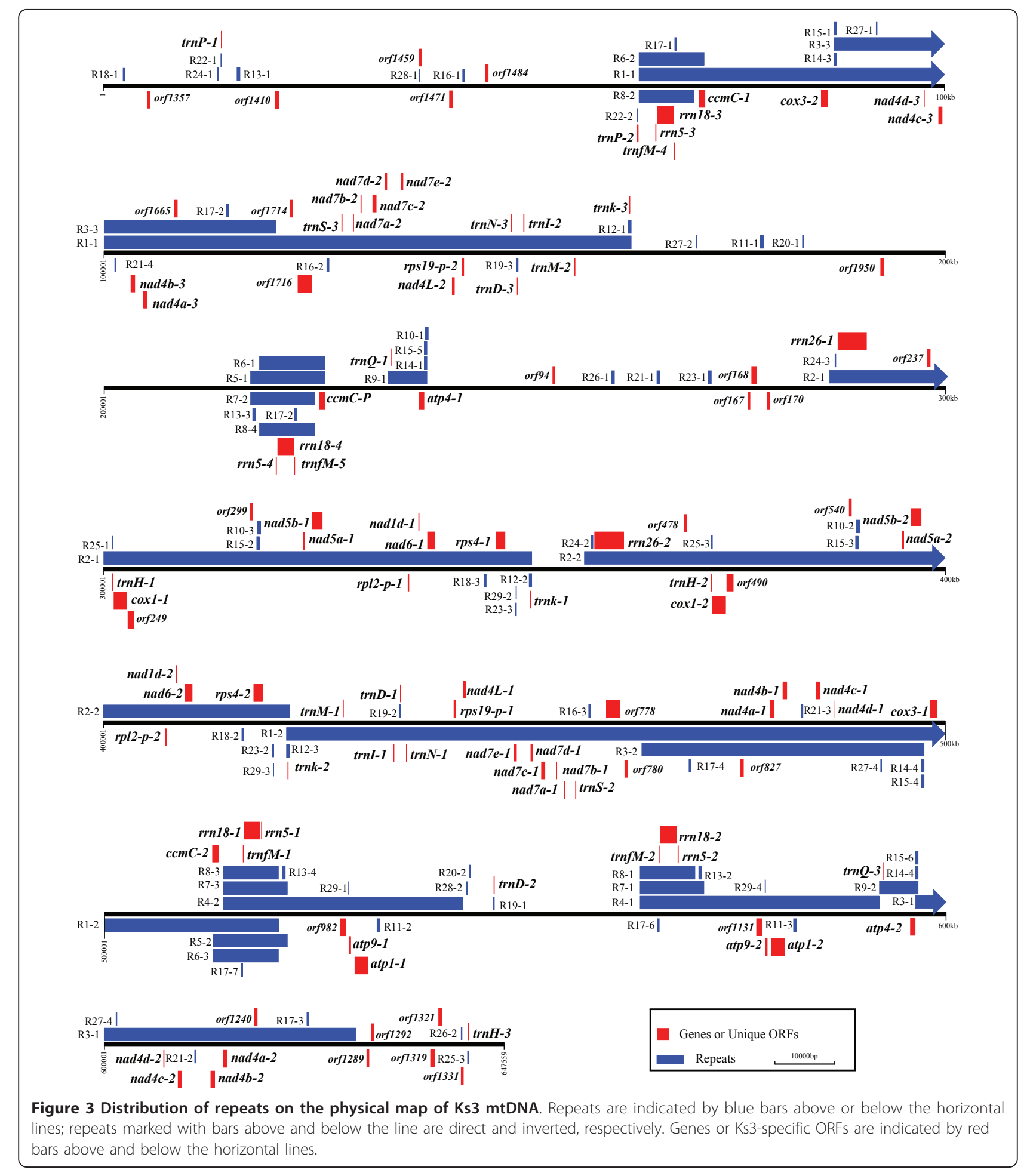

mtDNA sequence is almost identical to the previously reported sequence of $T$. aestivum cv. Chinese Spring, except for seven single nucleotide polymorphisms (SNPs) and 10 indels (insertions and deletions) [13,20]. As a result, $\mathrm{Km} 3 \mathrm{mtDNA}$ and Chinese Spring mtDNA have almost identical repeats (Additional File 9). We found that four repeats $(<500 \mathrm{bp})$ were almost identical in the two mitochondrial genomes; R12, R19, R20, and R22 in Ks3 mtDNA corresponded to R11, R15, R13, and $\mathrm{R} 14$ in $\mathrm{Km} 3 \mathrm{mtDNA}$. Ten repeats were specific to Ks3 
A

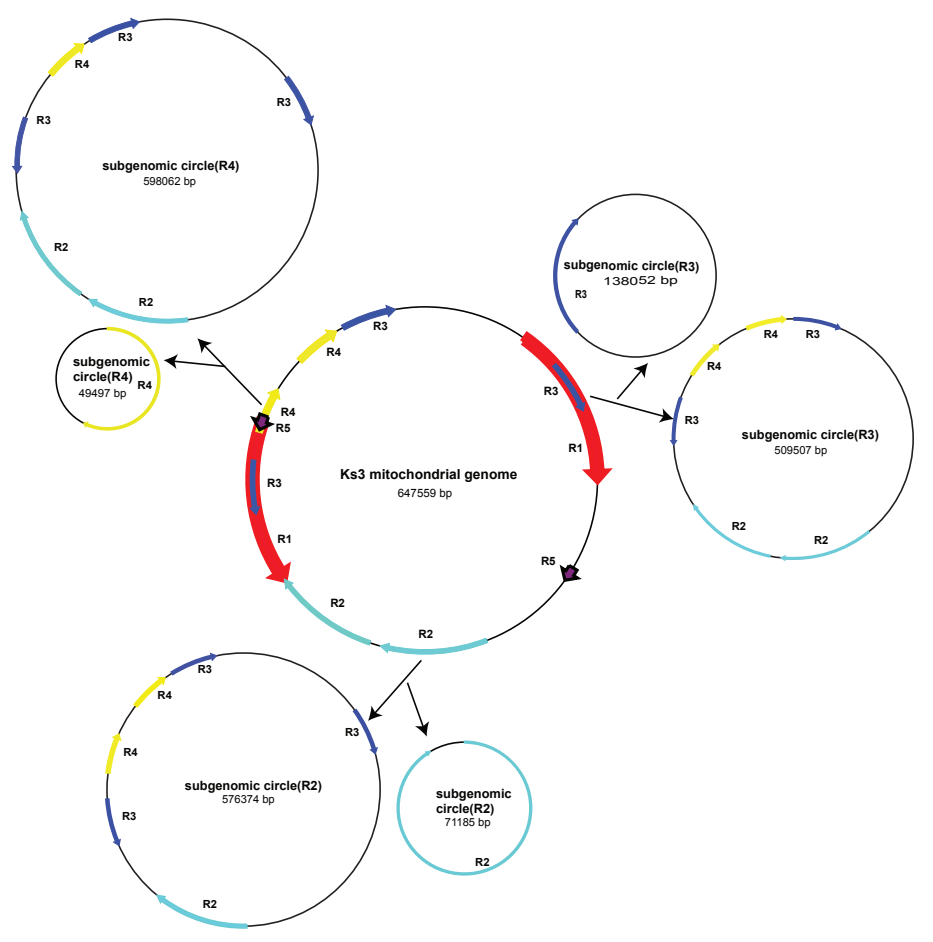

B

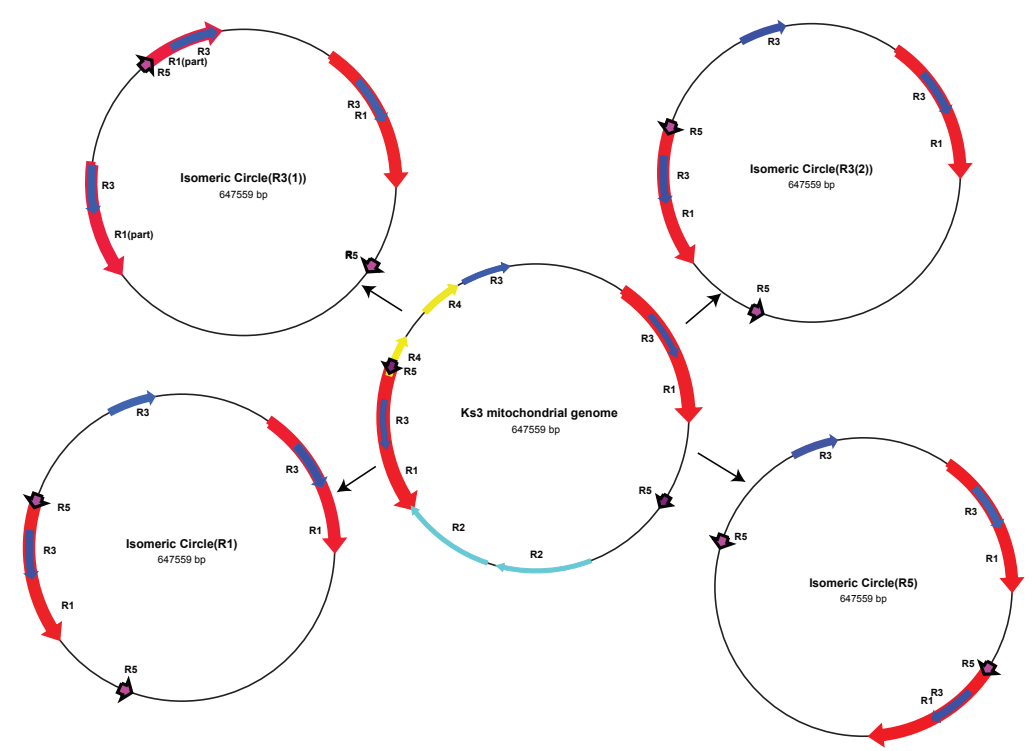

Figure 4 The predicted multipartite structures of Ks3 mtDNA. (A) Three pairs of subgenomic molecules produced by recombination of the DR pairs: $65 \mathrm{~kb}$ (R2), $33 \mathrm{~kb}$ (R3), and $28 \mathrm{~kb}$ (R4). (B) Four isomers of the MC molecule produced by recombination of the IR pairs: $99 \mathrm{~kb}$ (R1), 33 $\mathrm{kb}(\mathrm{R} 3), 33 \mathrm{~kb}(\mathrm{R} 3)$, and $8.8 \mathrm{~kb}(\mathrm{R} 5)$.

mtDNA and there were six specific repeats in $\mathrm{Km} 3$ mtDNA (Additional Files 10, 11). As shown in Additional File 11 the relationship between the large repeats in $\mathrm{Km} 3$ and $\mathrm{Ks} 3 \mathrm{mtDNA}$ is complicated. Four large repeats, R1, R2, R3, and R4, in Ks3 mtDNA were much bigger than the corresponding repeats in $\mathrm{Km} 3 \mathrm{mtDNA}$. $\mathrm{R} 2$ and $\mathrm{R} 8$ of $\mathrm{Km} 3$ showed homology to a fragment located at one end of R2 in Ks3 mtDNA. The two ends of R1 of $\mathrm{Km} 3$ were also homologous to the two ends of $\mathrm{R} 2$ of $\mathrm{Ks} 3$, whereas the central fragment of R1 of $\mathrm{Km} 3$ 
displayed no homology to the repeats of Ks3. A majority of R3 of $\mathrm{Km} 3$ was homologous to R4 of Ks3, but it was split in two locations.

\section{Protein-coding and RNA genes between $\mathrm{Ks} 3$ and $\mathrm{Km} 3$ mtDNAs}

The cytoplasm of $\mathrm{Km} 3$ and $\mathrm{Ks} 3$ originated from common wheat and Aegilops kotschyi belongs to two different genera, Triticum and Aegilops, respectively. Most of the protein-coding genes are highly conserved, especially in size, except atp6, nad6, nad9, and rps19-p (KM is prefixed to the names of genes/ORFs encoded in $\mathrm{Km} 3$ and KS is prefixed to those in Ks3; Additional File 12). For instance, the 5'-end of KSapt6 and KMatp6 is conserved but the 3'-end of KSapt6 is extended by $78 \mathrm{bp}$. Another example is nad9: due to deletion of four bases (TGTG) upstream of KSnad9, its ORF is 291 bp shorter than that of KMnad9. An extreme case is rpsl5, which is absent in Ks3 but present in Km3. A DNA exchange between mtDNA and nuclear DNA might have occurred, resulting in a nuclear rps 15 protein, if the rps 15 protein is proven to exist; otherwise, we have a defective Ks3 mitochondrial ribosome without rpl5.

We identified 32 SNPs scattered among 13 proteincoding genes: 12 were synonymous (KSapt1, KSmatR, KSrps 13, and KSnad4) and 20 were non-synonymous (Table 2). Most of these variations were actually transversions rather than the expected transitions. It is also remarkable that, when compared with $\mathrm{Km} 3$, many variations were found among ribosomal protein-coding genes, such as KSrps1, KSrps2, KSrps3, and KSrps4. These nonsynonymous changes in protein sequences are candidates for functional scrutiny in searching for molecular mechanisms of CMS, since protein-coding genes in plant mtDNA are extraordinarily conservative and their evolutionary rate is very low among different types of plants [6-8].

A majority of rRNA and tRNA genes were highly conserved between Ks3 and Km3 mtDNAs. Both, however, had missing sequences: $\mathrm{Ks} 3$ lost $\operatorname{trn} A$ and $\mathrm{Km} 3$ lost trnH. A similar case was also seen among rRNAs. For instance, Ks3 mtDNA did not include KSrrn26-p. Moreover, there were more genes and exons in Ks3 than in $\mathrm{Km} 3 \mathrm{mtDNA}$, as several large-sized repeats were unique to Ks3 mtDNA.

\section{ORFs between $\mathrm{Ks} 3$ and $\mathrm{Km} 3 \mathrm{mtDNAs}$}

Since novel ORFs may be relevant to CMS [21,22], we classified all possible ORFs in the $\mathrm{Km} 3$ and $\mathrm{Ks} 3$ mtDNA. We found 149 in Km3 and 248 in Ks3 with a length equal to or greater than $300 \mathrm{bp}$. The additional ORFs in Ks3 reflect the greater length of the Ks3 mtDNA. In addition to copy number and length variations, we also found some ORFs that were unique to Ks3, based on BLAST2 searches (Table 3 Figure 5).
Table 2 Differences in genes coding proteins between the mtDNA of $\mathrm{Km} 3$ and $\mathrm{Ks} 3$

\begin{tabular}{|c|c|c|c|c|}
\hline Gene & $\begin{array}{l}{\mathrm{Km} 3-\mathrm{Ks}^{\mathrm{a}}}^{\text {(nucleic acid) }}\end{array}$ & $\begin{array}{l}\text { Km3-Ks3 }{ }^{\mathrm{b}} \\
\text { (amino acid) }\end{array}$ & Mutation type $^{c}$ & SNP type \\
\hline $\operatorname{atp1}$ & 204T-G & & S & transversion \\
\hline \multirow[t]{3}{*}{ atp4 } & 249G-T & 84Glu-Pro & N & transversion \\
\hline & $250 G-C$ & 84Glu-Pro & N & transversion \\
\hline & $251 \mathrm{~A}-\mathrm{C}$ & 84Glu-Pro & N & transversion \\
\hline \multirow[t]{3}{*}{ ccmFN } & $306 C-A$ & & S & transversion \\
\hline & $906 \mathrm{~A}-\mathrm{C}$ & & S & transversion \\
\hline & $1103 \mathrm{~A}-\mathrm{T}$ & 368Gln-Leu & N & transversion \\
\hline \multirow[t]{2}{*}{$\operatorname{cox} 1$} & $1575 \mathrm{~A}-\mathrm{G}$ & & S & transition \\
\hline & 1456T-A & 486Cys-Ser & N & transversion \\
\hline \multirow[t]{3}{*}{$\cos 3$} & $157 A-C$ & 53lle-Leu & N & transversion \\
\hline & $687 \mathrm{G}-\mathrm{T}$ & 229Gln-His & N & transversion \\
\hline & $688 \mathrm{~A}-\mathrm{C}$ & 230Met-Leu & N & transversion \\
\hline matR & 1302T-G & & S & transversion \\
\hline nad3 & 185T-C & 62Leu-Pro & $\mathrm{N}$ & transition \\
\hline \multirow[t]{4}{*}{ nad4 } & $804 C-A$ & & S & transversion \\
\hline & $822 \mathrm{G}-\mathrm{A}$ & & S & transition \\
\hline & 1290G-T & & S & transversion \\
\hline & $1476 C-A$ & & S & transversion \\
\hline rps1 & $398 C-T$ & 133Thr-Ile & N & transition \\
\hline $\operatorname{rps} 13$ & $45 \mathrm{~A}-\mathrm{C}$ & & S & transversion \\
\hline \multirow[t]{6}{*}{ rps2 } & 837A-T & 280Gln-Lys & N & transversion \\
\hline & 838C-A & 280GIn-Lys & N & transversion \\
\hline & $845 G-C$ & 282Ser-Thr & $\mathrm{N}$ & transversion \\
\hline & 961C-A & 321His-Asn & $\mathrm{N}$ & transversion \\
\hline & 1002G-T & & S & transversion \\
\hline & 1062C-A & & $S$ & transversion \\
\hline \multirow[t]{4}{*}{ rps3 } & $256 C-A$ & 86Gln-Lys & $\mathrm{N}$ & transversion \\
\hline & $505 G-T$ & 169Asp-Phe & N & transversion \\
\hline & 505A-T & 169Asp-Phe & N & transversion \\
\hline & $1333 \mathrm{~A}-\mathrm{C}$ & 445Lys-Gln & N & transversion \\
\hline \multirow[t]{2}{*}{ rps4 } & 146G-T & 49Arg-Leu & $\mathrm{N}$ & transversion \\
\hline & $236 \mathrm{~T}-\mathrm{G}$ & 79Leu-Arg & $N$ & transversion \\
\hline
\end{tabular}

aLocation of base mutation.

bocation of amino acid mutation.

'S, Synonymous; N, Non-synonymous.

Among them, six (KSorf1289, KSorf170, KSorf1950, KSorf174, KSorf168, and KSorf982) were novel; a database search performed with the Blast network service using default parameters revealed no homologies to other sequences in the NCBI databases. Two ORFs (KSorf1292 and KSorf778), which were situated in two Ks3-specific mtDNA regions (U23 and U30), showed significant homology to wheat chloroplast DNA. As mentioned above, two Ks3 mtDNA fragments homologous to wheat ctDNA were unique and not found in Km3 mtDNA. This indicates that KSorf1292 and KSorf 778 were probably derived from an extraneous wheat chloroplast genome. Another pair of ORFs, KSorf1321 and KSorf1319, located in a Ks3 mtDNA 
Table 3 Unique ORFs of Ks3 mtDNA

\begin{tabular}{|c|c|c|c|c|c|c|}
\hline Predicted ORF $^{a}$ & $\begin{array}{l}\text { Ks3 MC coordinates } \\
\text { Copy-1 }\end{array}$ & Copy-2 & Copy-3 & Size (bp) & Sequence homology & Unique region $^{b}$ \\
\hline orf94 & $253294-253593$ & & & 300 & Two discrete segments of $\mathrm{Km} 3 \mathrm{mtDNA}$ & \\
\hline $\operatorname{orf1289*}$ & $631510-631202$ & & & 309 & None & U23 \\
\hline orf1331 & $642756-642442$ & & & 315 & Two discrete segments of $\mathrm{Km} 3 \mathrm{mtDNA}$ & \\
\hline orf299(orf540) & 317428-317745 & $388612-388929$ & & 318 & Partial homology to Km3 mtDNA & Partial U12 \\
\hline orf1459 & $37609-37929$ & & & 321 & Partial homology to $\mathrm{Km} 3 \mathrm{mtDNA}$ & Partial U37 \\
\hline $\operatorname{orf170*}$ & $279136-278813$ & & & 324 & None & U32 \\
\hline orf167 & $276799-276476$ & & & 324 & Partial homology to Km3 mtDNA & Partial U32 \\
\hline orf237(orf478) & $297847-298185$ & $369031-369369$ & & 339 & Partial homology to Km3 mtDNA & Partial U25 \\
\hline orf1357 & $5620-5276$ & & & 345 & Two discrete segments of $\mathrm{Km} 3 \mathrm{mtDNA}$ & \\
\hline orf1484 & $45478-45831$ & & & 354 & Two discrete segments of $\mathrm{Km} 3 \mathrm{mtDNA}$ & \\
\hline orf1292* & $631766-632140$ & & & 375 & Triticum aestivum chloroplast DNA & U23 \\
\hline $\operatorname{orf1321*}$ & $639777-640151$ & & & 375 & Secale cereale mitochondrial pol-r gene & U36 \\
\hline orf1240(orf1665;orf827) & $617878-618261$ & 108375-108758 & $476066-475683$ & 384 & Partial homology to Km3 mtDNA & Partial U21 \\
\hline orf1950* & 192718-192320 & & & 399 & None & U38 \\
\hline orf780(orf1714) & $462346-461939$ & $122095-122502$ & & 408 & Partial homology to Km3 mtDNA & Partial U31 \\
\hline orf1471* & $41604-41194$ & & & 411 & None & U37 \\
\hline orf1410 & $20945-20457$ & & & 489 & Two discrete segments of $\mathrm{Km} 3 \mathrm{mtDNA}$ & \\
\hline orf1319* & 639287-638796 & & & 492 & Secale cereale mitochondrial pol-r gene & U36 \\
\hline orf168* & $276925-277623$ & & & 699 & None & U32 \\
\hline orf982(orf1131)* & $528726-528013$ & $578221-577508$ & & 714 & None & U31 \\
\hline orf249(orf490)* & 303665-302904 & $374849-374088$ & & 762 & orf256 & U17 \\
\hline orf778(orf1716) & $459748-461400$ & $124693-123041$ & & 1653 & $\begin{array}{l}\text { Triticum aestivum chloroplast DNA } \\
\text { psaA gene }\end{array}$ & Partial U30 \\
\hline
\end{tabular}

aoldface: ORFs that are homologous to wheat ctDNA.

${ }^{b}$ ORFs are entirely or partially located in the corresponding unique regions of $\mathrm{Km} 3 \mathrm{mtDNA}$.

*These ORFs do not show significant homology to Km3 mtDNA.

unique region, U36, exhibited homology to a DNA polymerase in rye mtDNA. This result indicates that KSorf1321 and KSorf1319 likely encode proteins with similar function in Ks3 mtDNA, but further empirical data are needed. It is notable that KSorf 249 in Ks3 mtDNA is homologous to orf256, a candidate for a sterile gene associated with wheat T-CMS, which originated from the transfer of the wheat nuclear counterpart into Triticum timopheevii cytoplasm. In wheat T-CMS, the chimeric gene orf256 is situated upstream of $\operatorname{cox} 1$, is transcribed together with cox 1 , and expresses a $7-\mathrm{kDa}$ protein that is not found in fertile lines $[23,24]$. Our data showed that KSorf249 resembled orf256 upstream of KScox 1 , and deserves further study. Previous studies have shown that the ORFs involved in CMS are usually located in the vicinity of known genes or form a chimeric gene by overlapping with parts of known genes in the plant mitochondrial genome. For example, urf13-T which leads to CMS in maize T-CMS is located downstream of atp6, which provides the regulatory sequence, and the two are co-transcribed [25]. Similarly, orf107, the CMS gene in sorghum A3-CMS, forms a chimeric sequence by partially overlapping the 5 -end with that of atp9 [26,27]. Some of the Ks3-specific KSorfs have similar structures to known ORFs involved in CMS in other plant mtDNAs (Figure 5A, B).

We also categorized Ks3-specific ORFs into two basic groups: those that were partially homologous to $\mathrm{Km} 3$ mtDNA (Figure 5C) and those that were almost entirely homologous to Km3 mtDNA (Figure 5D). Partial segments of seven ORFs (KSorf299, KSorf1459, KSorf167, KSorf237, KSorf1240, KSorf780, and KSorf778) were located in corresponding Ks3-specific regions, whereas another sequence of these ORFs was homologous to Km3 mtDNA (Table 3). In addition, with the exception of KSorf167, these ORFs were located in Ks3 mtDNA repeat regions (Figure 3). As shown in Figure $5 B$ and $5 D$, five ORFs (KSorf1357, KSorf94, KSorf1331, KSorf1410, and KSorf1484) had remarkable homology to Km3 mtDNA, but homologous $\mathrm{Km} 3 \mathrm{mtDNA}$ were divided into two discrete segments in the $\mathrm{Km} 3$ mitochondrial genome, which indicates that they are likely derived from different parts of $\mathrm{Km} 3 \mathrm{mtDNA}$. It is notable that five ORFs were not situated in repeat regions of Ks3 mtDNA (Figure 3).

\section{Comparison among angiosperm mtDNAs}

We used MultiPipMaker to align similar regions in two or more DNA sequences using one of the DNA 


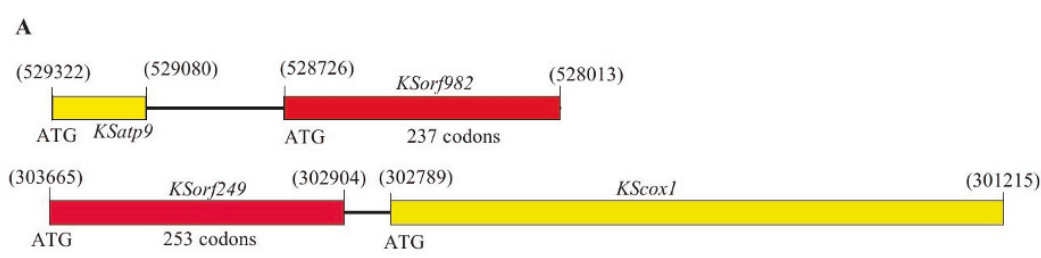

B

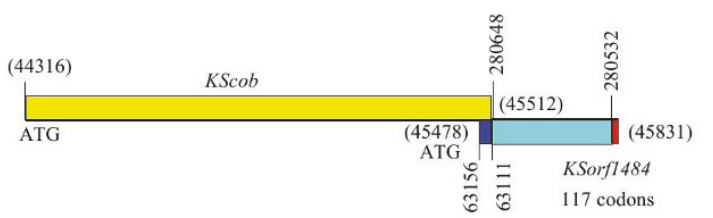

$\square$ Segments of Ks3 mtDNA homo-

logous to $\mathrm{Km} 3$

Unique segments of Ks3 mtDNA

Known genes of Ks3 mtDNA

C

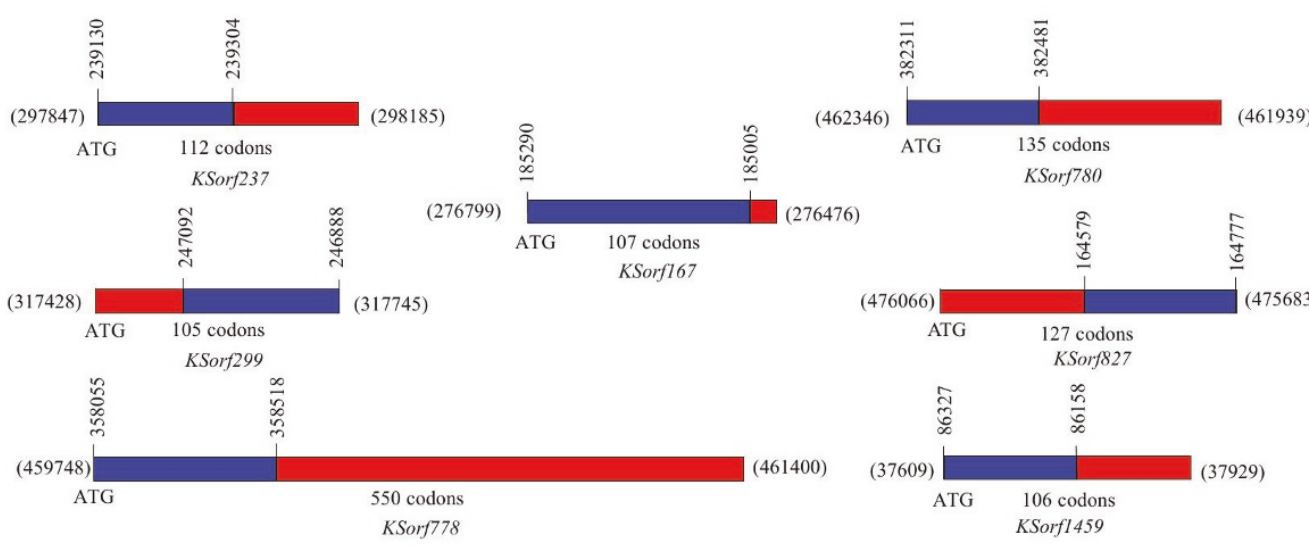

D
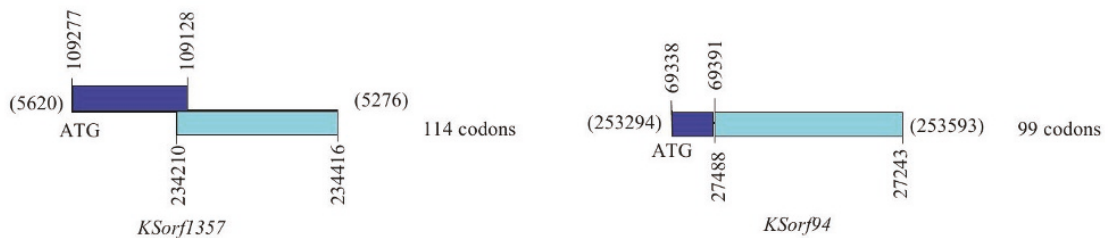

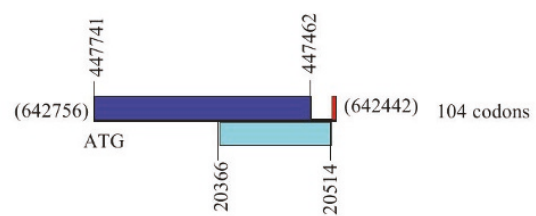

KSorf1331

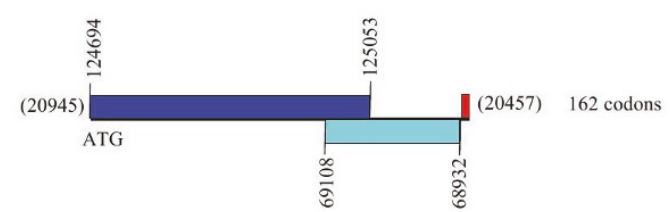

KSorf1410

Figure 5 Unique ORFs of Ks3 mtDNA. (A) ORFs located in the vicinity of known genes; (B) ORFs overlapping with known genes; (C) partial sequences of ORFs that are homologous to Km3 mtDNA; (D) ORFs that are homologous to two discrete segments of Km3 mtDNA. Red bars indicate the unique sequences of $\mathrm{Ks} 3 \mathrm{mtDNA}$. Blue bars and blue-green bars indicate the homology of Ks3 mtDNA to Km3 mtDNA. Yellow bars indicated known genes of $\mathrm{Ks} 3 \mathrm{mtDNA}$. The vertical numbers show the coordinates of the homologous fragments of ORFs in the Km3 mtDNA MC molecule. The numbers in parentheses indicate the coordinates of ORFs in the Ks3 mtDNA MC molecule. 
sequences as a reference; in our study, Ks3 mtDNA was used as the reference unless stated otherwise. Comparing Ks3 mtDNA to those of Km3, rice, maize, Arabidopsis thaliana, and rapeseed (Additional File 13), we noticed several interesting features. First, the alignable Ks3 sequence $(87.6 \%)$ was $83 \%$ identical to that of $\mathrm{Km} 3$ mtDNA. For a more distant sequence comparison, only $34.6 \%$ and $32.2 \%$ of the Ks3 mtDNA matched those of maize and rice with an identity of more than $78 \%$, respectively. Only $15.6 \%$ and $15.5 \%$ of the Ks 3 mtDNA was shared with Arabidopsis thaliana and rapeseed, at more than $76 \%$ identity, respectively, and the longest fragment was only $2 \mathrm{~kb}$. Nevertheless, due to greater evolutionary pressure, coding sequences in angiosperm mtDNA are more conservative, whereas the non-coding parts are highly divergent (Additional File 13) $[9,28]$.

We also compared the copy number of mitochondrial genes among Ks3, Km3, maize, and rice (Additional File 14). Ks3 mtDNA appeared to have the most multi-copy genes, in contrast to $\mathrm{Km} 3 \mathrm{mtDNA}$, in which only atp6 and atp 8 had two copies. Ribosomal protein-coding genes and $\operatorname{trn} A$ genes appeared to be more divergent among angiosperm mtDNAs. For example, rice and $\mathrm{Km} 3 \mathrm{mtDNAs}$ contained rpl5 but maize and Ks3 mtDNAs did not. KSrpl2, KSrps19, KMrpl2, and KMrps19, as truncated pseudogenes, were not complete ORFs, whereas rpl2 and rps 19 in rice mtDNA included complete ORFs; however, maize mtDNA did not include these sequences. Moreover, $\mathrm{Km} 3$ and maize possessed $\operatorname{trn} A$ in their mtDNAs, but Ks3 and rice did not.

We also compared gene order in Ks3 mtDNA to that in $\mathrm{Km} 3$, maize, and rice mtDNA, excluding tRNA genes (Additional File 15). First, $r r n 5$ and $r r n 18$ were inserted into the nad5c-nad1e-matR-rps1-ccmFN cluster shared by other grass mtDNAs to form a new cluster unique to $\mathrm{Ks} 3$ and $\mathrm{Km} 3 \mathrm{mtDNAs}$. Second, 11 clusters were found to be syntenic in Ks3 and Km3 mtDNAs. Third, Ks3 mtDNA shared four two-gene clusters, rrn5-rnn18, nad3-rps12, rps13-nad1bc, and nad9-nad2cde, with rice and maize mtDNAs. Fourth, Ks3 mtDNA shared four two-gene clusters, rps3a-rpl16, rnn26-cox1, nad6-rps4 and $a t p 1-\operatorname{cox} 2 a b$, with maize alone. Fifth, three other two-gene clusters, rpl16-rps3b, nad4l-rps19, and nad5ab-rpl2, were common only to Ks3 and rice mtDNAs but not to maize mtDNA. These variations in gene order were readily identified by syntenic analysis.

\section{Discussion}

\section{Comparative analysis of $\mathrm{Km} 3$ and $\mathrm{Ks} 3 \mathrm{mtDNAs}$}

The Ks3 MC molecule was $192 \mathrm{~kb}$ larger than that of $\mathrm{Km} 3$; Ks3 had additional long repeat elements-four of them were more than $20 \mathrm{~kb}$ in size-and the longest repeat was $98,977 \mathrm{bp}$ in length. Similar results were also reported in TK18-MS, a cytoplasmic male sterile line of sugar beet, which contains a pair of repeats of $86,816 \mathrm{bp}$ in its MC molecule [29]. Although repeat content of mtDNA can account for more than $30 \%$ of total genome sequence length, as in indica rice 93-11, where repeats greater than $2 \mathrm{~kb}$ in size constitute $27.7 \%$ of the total mtDNA [30], the size of mtDNAs of cytoplasmic male sterile lines seems to be dramatically larger than that of maintainer lines. The intergenic region of plant mtDNAs often contains retrotransposons transferred from nuclear and chloroplast genomes [31,32]. Ks3 mtDNA again had more retrotransposons than Km3: 12 vs. 5. However, the percentages of these retrotransposons in both $\mathrm{Ks} 3$ and $\mathrm{Km} 3$ were not as high as in maize mtDNA, where retrotransposons account for $4.44 \%$ of the total genome [33], but where the rate of gene transfer is generally deemed low [34].

Frequent recombination events have distorted the synteny between $\mathrm{Ks} 3$ and $\mathrm{Km} 3 \mathrm{mtDNAs}$ (Figure 6), as also seen among other plant mtDNAs [35]. Ks3 mtDNA had $11.38 \%$ unique sequences when compared to $\mathrm{Km} 3$ mtDNA; $7.3 \%$ of Ks3 mtDNA sequences are novel but most of these are located in intergenic regions that

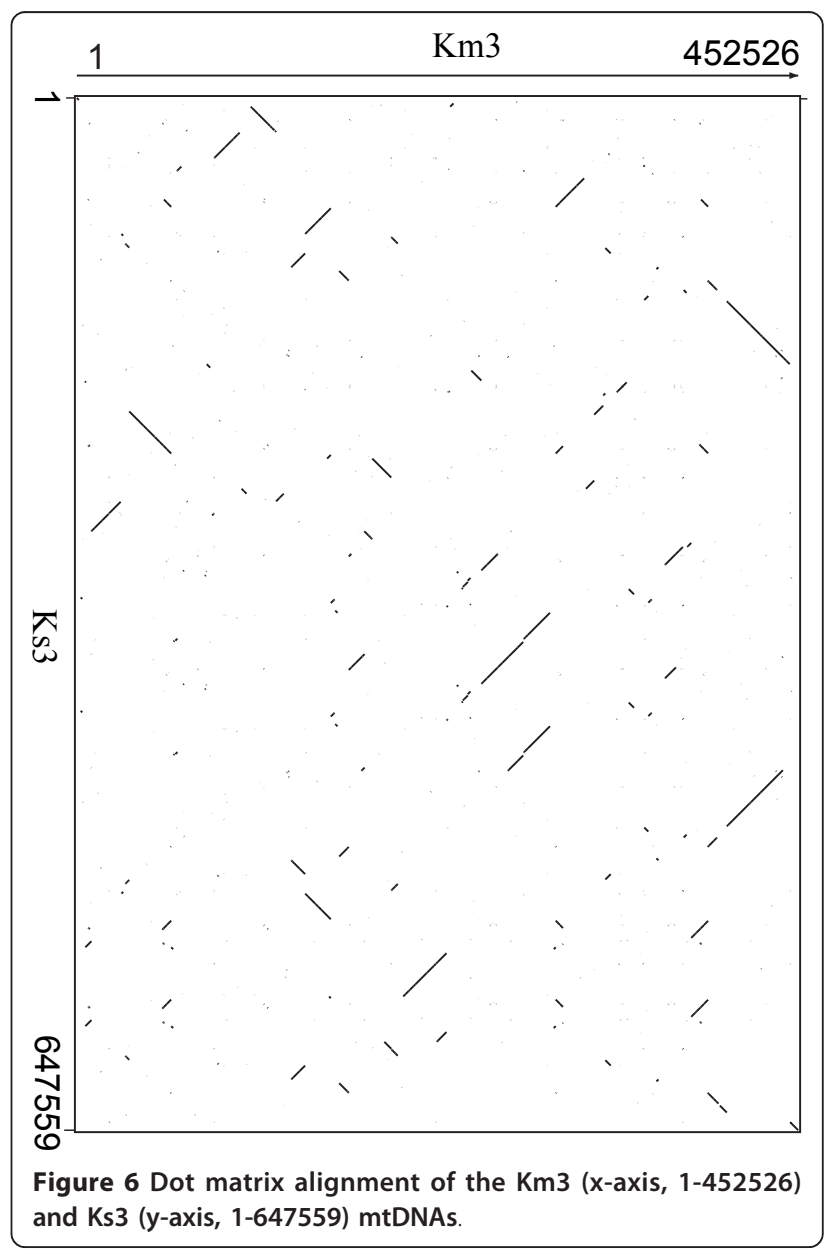


show a faster rate of evolution [9,28]. Furthermore, although many gene sequences were highly conserved between the two genomes, there were exceptions. $r p l 5$ was missing in $\mathrm{Ks} 3$, and the sequences of atp6, nad9, and nad 6 between $\mathrm{Km} 3$ and $\mathrm{Ks} 3 \mathrm{mtDNAs}$ were very different. In addition, the number of SNPs between the $\mathrm{Ks} 3$ and $\mathrm{Km} 3 \mathrm{mtDNAs}$ was also significant, compared to those in a CMS line of sugar beet (Owen CMS), which has 24 SNPs in 11 protein-coding genes compared to the fertile form [29]. Finally, there were 22 ORFs unique to Ks3 mtDNA. These differences in protein-coding sequences between $\mathrm{Ks} 3$ and $\mathrm{Km} 3 \mathrm{mtDNAs}$ are good candidates for contributing to the CMS phenotype.

\section{Structural diversity among plant mtDNAs}

Our analysis of structural diversity is necessary to understanding the sequence diversity among plant mtDNAs [34]. We detected 29 repeats of more than 100 bp in Ks3 mtDNA, including direct repeats (DR) and inverted repeats (IR), and their roles in shaping subgenomic and isomeric structures in Ks3 mtDNA are of importance. It is believed that proteins encoded by nuclear genes are involved in mismatch repair and recombination of mtDNAs. A gene, Msh1, in the nuclear genome homologous to the Escherichia coli MutS mismatch repair component, $\operatorname{Rec} A 3$, affects structural diversity in $A$. thaliana [10]. In maize, the P2 nuclear genotype is used as a system for understanding mutations in mtDNA, where abnormal recombination products remarkably increase as the copy number of subgenomic molecules of maize mtDNA increases [36]. Research has shown that when the gene homologous to Msh1 in tobacco and tomato is knocked out by RNAi, novel mitochondrial genome organizations are observed, and plants show a male sterility phenotype [37].

\section{Molecular mechanisms of wheat K-type CMS}

We conducted extensive sequence comparison between $\mathrm{Ks} 3$ and $\mathrm{Km} 3 \mathrm{mtDNAs}$ to search for functional alterations of genes that were responsible for the CMS phenotype in plants. We noticed that Ks3 mtDNA encodes several partial subunits of the respiratory chain complex, including ATP4, ATP6, NAD3, NAD6, NAD9, COX1, and COX3 (Additional File 16). Any of these altered proteins may interfere with the normal function of respiratory chain reactions, weakening energy supplies and stalling pollen development [38]. In addition, we also observed amino acid variations among RPS1, RPS2, RPS3, RPS4, and ccmFN, as well as a missing RPL5 in $\mathrm{Ks} 3 \mathrm{mtDNA}$. Whether these variations are related to wheat K-type CMS requires further study.

Research on the expression of novel ORFs in $\mathrm{Ks} 3$ mtDNA is also necessary [39], as the relevance of unknown ORFs to CMS has been reported, such as urf13- $T$ in maize [40], orf 224 and orf222 in rapeseed [41], orf522 in sunflower [42], orf138 in radish [43], orf107 in sorghum [26], and orf79 in rice [21]. The proteins encoded by these ORFs involved in CMS may have structures similar to ATP synthese subunits, which would lead to functional competition; $p c f$ in petunia [44] and orf456 in pepper [45] were shown to be involved in recombination with the genes encoding cytochrome oxidase $(\operatorname{cox} 1$ and $\operatorname{cox} 2)$. Another functional scenario is that these novel ORFs may be involved in CMS by damaging mitochondrial membrane structure. In maize, URF13, encoded by T-urf13, assembles into a tetramer that penetrates the mitochondrial membrane, and the resulting permeability change affects normal mitochondrial function [46,47].

Previous studies have shown that the process of anther abortion in K-type CMS occurs in the two-cell stage or the late period of the three-cell state of anther development, and the development of pollen is regulated by multiple genes [48]. Therefore, it is necessary to profile the expression of the CMS-specific ORFs in distinct developmental stages, including the microspore mother cell, tetrad, single cell pollen grains, two-cell pollen grains, and three-cell pollen grains. We are preparing to explore molecular mechanisms of wheat K-type CMS through a combination of genomic and proteomic tools, such as the analysis of the transcription and function of the unique ORFs found in Ks3 mtDNA.

\section{Conclusion}

The complete mitochondrial genome of the wheat K-type CMS line Ks3 is very different from that of its maintainer line, $\mathrm{Km} 3$, especially in non-coding sequences. The Ks3 mtDNA is $647,559 \mathrm{bp}$ and harbors 34 known protein-coding genes, three rRNAs (18 S, $26 \mathrm{~S}$, and $5 \mathrm{~S}$ rRNAs), 16 different tRNAs, Ks3-specific mtDNA (> $100 \mathrm{bp}, 11.38 \%$ ), and repeats (> $100 \mathrm{bp}$, 29 units). In addition, $r p l 5$ is missing, and 32 SNPs are involved in 13 protein-coding, albeit functionally irrelevant, genes, and 22 ORFs are unique in Ks3. All these sequence variations are candidates for CMS. Comparative analysis of the mtDNA of several angiosperms including $\mathrm{Ks} 3, \mathrm{Km} 3$, rice, maize, Arabidopsis thaliana, and rapeseed, indicates that non-coding sequences are the most frequently reorganized part of the mitochondrial genome during mtDNA evolution in higher plants.

\section{Methods}

\section{Plant materials}

A wheat CMS line with male-sterile cytoplasm from Aegilops kotschyi was designated as K-type Yumai 3 CMS line (abbreviated Ks3), and its isonuclear line with normal male-fertile cytoplasm was designated as K-type 
Yumai 3 (Triticum aestivum cv. Yumai 3) maintainer line (abbreviated $\mathrm{Km} 3$ ) [13]; both were harvested from winter crops in Henan Province, China.

\section{Mitochondrial DNA extraction}

Mitochondria were isolated from etiolated 2-week-old seedlings of $\mathrm{Km} 3$ and $\mathrm{Ks} 3$ according to a previously published procedure [15]. Mitochondrial fractions were collected by differential centrifugation, incubated with DNase I for $1 \mathrm{~h}$ on ice to eliminate linear DNA, and further purified by centrifugation in a discontinuous sucrose-density gradient $(1.2 \mathrm{M} / 1.6 \mathrm{M} / 2.0 \mathrm{M})$. The purified mitochondria band was carefully collected from the 1.6 M/1.2 M interface and washed with $0.4 \mathrm{M}$ sucrose. The fraction was finally lysed in 2\% Sarkosyl for mtDNA extraction, followed by phenol-chloroform extraction and ethanol precipitation.

\section{Genome library construction and sequencing}

Mitochondrial genome BAC libraries for $\mathrm{Ks} 3$ and $\mathrm{Km} 3$ were constructed following a previously published procedure with minor modifications [49]. Mitochondria genomic DNA was partially digested with Sau3AI, sizefractioned by pulsed-field gel electrophoresis, and ligated to PIndigoBAC-5 BamHI cloning-ready vector (Epicentre Biotechnologies, Madison, WI, USA; http://www. epibio.com). The ligation mix was transformed into DH10B-competent cells through electroporation. Highdensity nylon filters (eight 384-well plates) were screened for a tiling path that covers the entire genome. Shotgun plasmid libraries were made from minimal tiling clones in the pUC-18 vector, and used for sequencing on ABI-3730xl DNA analyzers.

\section{Analysis of sequence data}

The entire nucleotide sequences of $\mathrm{Km} 3 \mathrm{mtDNA}$ (accession number EU534409) and Ks3 mtDNA (accession number GU985444) were determined at the Beijing Institute of Genomics, Chinese Academy of Sciences, and DNA sequences were assembled using the software package phred/phrap/consed [50,51] on a PC/UNIX platform. Physical gaps were closed based on direct sequencing of selected clones. The final assembly of Ks3 mtDNA and Km3 mtDNA included 11,200 and 9931 sequences, respectively. Both genome sequences have nine-fold coverage on average, with a quality value Q20. The final master circle (MC) molecules were obtained with manual editing.

The mitochondrial sequences were annotated with Glimmer 3.0 and BLAST tools, and tRNA genes and their secondary structures were identified according to tRNA scan-SE [52]. The Pairwise BLAST program on our local server was used for comparison between $\mathrm{Ks} 3$ mtDNA and $\mathrm{Km} 3 \mathrm{mtDNA}$ and $\mathrm{Ks} 3 \mathrm{mtDNA}$ and the mitochondrial genomes of other plants, with an E-value cutoff at 0.001. A database search was executed using the BLAST network service http://blast.ncbi.nlm.nih. gov/Blast.cgi with default parameters.

Alignments were obtained using MultiPipMaker, a web-based tool for genomic sequence alignments http://bio.cse.psu.edu/pipmaker [53,54]. The annotated Ks3 mtDNA genomic sequence was used as a reference genome and compared with mtDNA sequences from Km3 (Triticum aestivum cv. Yumai 3; EU534409), rice (AB076665, AB076666), maize NB (Zea mays ssp. Mays cytotype NB; AY506529), Arabidopsis (Arabidopsis thaliana; NC001284), and rapeseed (Brassica napus L.; AP006444)

\section{Additional material}

Additional file 1: Genes in the Ks3 mitochondrial genome. The file contains the list of size and MC coordinates of genes in the Ks3 mitochondrial genome, including Known protein-coding genes, rRNAs genes and tRNAs genes.

Additional file 2: Transposons in the Ks3 mitochondrial genome. The file contains the list of size and MC coordinates of transposons in the Ks3 mitochondrial genome. These transposons were identical to known those of rice or wheat with different identity, respectively.

Additional file 3: List of $\mathrm{Ks} 3 \mathrm{mtDNA}$ unique regions (compared with Km3 mtDNA). Compared with Km3 mtDNA, Ks3 mtDNA has 38 specific regions. The file contains the list of size and $M C$ coordinates of those $\mathrm{Ks} 3$-specific mtDNA regions. Some unique regions in $\mathrm{Ks} 3 \mathrm{mtDNA}$ were homologous to previously determined sequences in NCBI databases, while others could not be detected in NCBI databases.

Additional file 4: Map of homologous fragments in Ks3 mtDNA unique regions. These fragments homologous to sequences in NCBI databases mapped to Ks3-specific mtDNA regions. Black bars show Ks3 mtDNA unique regions, and fragments of Ks3 mtDNA unique regions homologous to NCBI databases are indicated by broad red bars. The vertical numbers show the coordinates of the homologous fragments in Ks3 mtDNA unique regions. The letters $A, B$, and $C$ indicate different annotated sequences in NCBI databases, shown in Additional File 3.

Additional file 5: List of Ks3 mtDNA sequences showing homology to ctDNA sequences. The file contains the list of size and MC coordinates of $\mathrm{Ks} 3 \mathrm{mtDNA}$ sequences showing homology to ctDNA sequences with more than $81 \%$ identity and a size range of 24 to 2790 bp.

Additional file 6: List of Ks3 mtDNA sequences of more than 100 bp showing homology to ctDNA sequences. The file contains the list of Ks3 mtDNA sequences of more than 100 bp showing homology to ctDNA sequences. These Ks3 mtDNA sequences showed homology to the ctDNA sequences bringing the corresponding chloroplast genes.

Additional file 7: List of wheat ctDNA sequences uniquely homologous to $\mathrm{Km} 3 \mathrm{mtDNA}$ and those uniquely homologous to Ks3 mtDNA. The file contains the list of size and MC coordinates of wheat ctDNA sequences uniquely homologous to Km3 mtDNA and those uniquely homologous to Ks3 mtDNA with different identity.

Additional file 8: Dot matrix representation of the Ks3 MC molecule. Repeats of more than $100 \mathrm{bp}$ are marked on the map. Red and blue dots and lines represent direct and inverted repeats, respectively. Nine repeats of more than 500 bp, R1-R9, are marked with arrows.

Additional file 9: List of repeats larger than $100 \mathrm{bp}$ found in $\mathrm{Km} 3$ mtDNA. The file contains the list of type, size and MC coordinates of 16 repeats larger than $100 \mathrm{bp}$ in $\mathrm{Km} 3 \mathrm{mtDNA}$. 
Additional file 10: Homology of mtDNA repeats between Ks3 and $\mathrm{Km} 3$. The file contains the list of homology of mtDNA repeats between $\mathrm{Ks} 3$ and $\mathrm{Km} 3$. Four repeats were almost identical in the Ks3 mtDNA and $\mathrm{Km} 3 \mathrm{mtDNA}$, while the relationship between the large repeats in two mitochondrial genomes is complicated.

Additional file 11: Comparison of mtDNA repeats between Ks3 and Km3. (A) Blue bars above or below the horizontal lines indicate one direct or inverted copy of repeats of Ks3 mtDNA, respectively. Netted bars show repeats of $\mathrm{Km} 3 \mathrm{mtDNA}$ homologous to $\mathrm{Ks} 3 \mathrm{mtDNA}$, and numbers in brackets indicate coordinates of partial homologous fragments in repeats of $\mathrm{Km} 3 \mathrm{mtDNA}$. Red bars indicate that the partial fragments in repeats of $\mathrm{Km} 3 \mathrm{mtDNA}$ did not show significant homology to repeats of $\mathrm{Ks} 3 \mathrm{mtDNA}$. (B) The specific mtDNA repeats of $\mathrm{Km} 3$ and $\mathrm{Ks} 3$ and mtDNA repeats shared by Ks3 and Km3. A pair of identical repeats is indicated by a left-right arrow.

Additional file 12: Alignments of atp6 (A), nad6 (B), nad9 (C), and rps19-p (D) between Ks3 and Km3. the file contains the alignments of atp6 (A), nad6 (B), nad9 (C), and rps19-p (D) between Ks3 and Km3. The identical sequences are highlighted with a dark background. The vertical arrow in (D) indicates deletion in Ks3 mtDNA (Ks3_seq) and STOP shows the location of the terminal codon (TAG)

Additional file 13: MultiPipMaker analysis of the mtDNA of several angiosperms. Ks3 mtDNA was used as the reference genome for comparison with those of $\mathrm{Km} 3$, rice, maize, Arabidopsis thaliana, and rapeseed. (A) Positions of genes or exons of Ks3 mtDNA are indicated with black bars, and their orientations are shown with arrows. The percentage identity is shown at the right side. (B) A schematic representation of (A) in which only representative genes of Ks 3 mtDNA are marked. Red and green bars indicate identity with $\mathrm{Km} 3 \mathrm{mtDNA}$ and other mtDNAs, respectively.

Additional file 14: The number of copies of mitochondrial genes in $\mathrm{Ks} 3, \mathrm{Km} 3$, maize, and rice. The file contains the list of the number of copies of mitochondrial genes in $\mathrm{Ks} 3, \mathrm{Km} 3$, maize, and rice. The identity of genes between $\mathrm{Ks} 3 \mathrm{mtDNA}$ and $\mathrm{Km} 3 \mathrm{mtDNA}$ was also shown.

\section{Additional file 15: Correlation of gene order between the} mitochondrial gene maps of $\mathrm{Ks} 3$ and $\mathrm{Km} 3(\mathrm{~A})$, maize (B), and rice (C). The file contains the comparison of gene order in Ks3 mtDNA to that in $\mathrm{Km3}$, maize, and rice mtDNA. The protein-coding and rRNAcoding genes are arranged from top to bottom for $\mathrm{Ks} 3$, and from left to right for Km3, rice, and maize, based on their order in the respective gene maps. Genes of $\mathrm{Km} 3$, rice, and maize are indicated by code numbers representing the corresponding $\mathrm{Ks} 3$ genes on the left margin of figures.

Additional file 16: Alignments of ATP6, NAD6, and NAD9 between $\mathrm{Km} 3$ and Ks3. the file contains the alignments of (A) KMATP6 and KSATP6, (B) KMNAD6 and KSNAD6, and (C) KMNAD9 and KSNAD9. The identical sequences are highlighted with a dark background.

\section{Abbreviations}

CMS: cytoplasmic male sterility; mtDNA: mitochondrial DNA; ctDNA: chloroplast DNA; MC: master circle; DR: direct repeats; IR: inverted repeats; ORF: open reading frame; atp 1, atp4, atp6, atp8, and atp9: ATP synthase subunits 1, 4, 6, 8, and 9 genes; cob: apocytochrome b gene; cox1-3: cytochrome $c$ oxidase subunits 1-3 genes; nad1-7, 9 and nad4L: NADH dehydrogenase subunits $1-7,9$, and $4 \mathrm{~L}$ genes; rpl2-p, rp/5, rp/16, rps $7-4, r p s 7$, rps 12-13, and rps 19-p: ribosomal protein large and small subunit genes; rrns and $r r n L$ : small and large subunit ribosomal RNA (rRNA) genes; trnX: transfer RNA (tRNA) genes, where $X$ is the one-letter abbreviation of the corresponding amino acid; SNPs: single nucleotide polymorphisms; aa: amino acids; nt: nucleotides; bp: base pairs; kb: kilobase.

\section{Acknowledgements}

This work was supported by the National Natural Science Foundation of China (30971668 and 30471081).

\section{Author details}

'The State Key Laboratory of Plant Cell and Chromosome Engineering, Institute of Genetics and Developmental Biology, Chinese Academy of
Sciences, Beijing, 100101, China. ${ }^{2}$ The CAS Key Laboratory of Genome Sciences and Information, Beijing Institute of Genomics, Chinese Academy of Sciences, Beijing, 100029, China. ${ }^{3}$ Agronomy College of Henan Agricultural University, Zhengzhou, 450002 , China. ${ }^{4}$ College of Biological Sciences of China Agricultural University, Beijing, 100094, China. ${ }^{5}$ Graduate University of Chinese Academy of Sciences, Beijing, 100049, China.

\section{Authors' contributions}

$\mathrm{HL}, \mathrm{PC}, \mathrm{QL}, \mathrm{GZ}, \mathrm{WY}$, and $\mathrm{DL}$ carried out the molecular experiments. $\mathrm{KZ}, \mathrm{XG}$ $\mathrm{SH}, \mathrm{JY}$, and $\mathrm{AZ}$ designed and coordinated all experiments. $\mathrm{HL}, \mathrm{PC}, \mathrm{QL}$, and FD performed the genomic analyses. All authors contributed to the manuscript and then read and approved the final version.

Received: 24 March 2010 Accepted: 29 March 2011

Published: 29 March 2011

\section{References}

1. Margulis $L$, Bermudes D: Symbiosis as a mechanism of evolution: status of cell symbiosis theory. Symbiosis 1985, 1:101-124.

2. Gray MW: Evolution of organellar genomes. Curr Opin Genet Dev 1999, 9:678-687.

3. Palmer JD, Herbon LA: Unicircular structure of the Brassica hirta mitochondrial genome. Curr Genet 1987, 11:565-570

4. Ward BL, Anderson RS, Bendich AJ: The mitochondrial genome is large and variable in a family of plants (Cucurbitaceae). Cell 1981, 25:793-803.

5. Schuster W, Brennicke A: The plant mitochondrial genome:physical structure, information content, RNA editing, and gene migration to the nucleus. Annu Rev Plant Physiol Plant Mol Biol 1994, 45:61-78.

6. Gray MW, Burger G, Lang BF: Mitochondrial evolution. Science 1999, 283:1476-1481.

7. Mackenzie S, Mcintosh L: Higher plant mitochondria. Plant Cell 1999, 11:571-586.

8. Knoop V: The mitochondrial DNA of land plants: peculiarities in phylogenetic perspective. Curr Genet 2004, 46:123-139.

9. Palmer JD, Adams KL, Cho Y, Parkinson CL, Qiu YL, Song K: Dynamic evolution of plant mitochondrial genomes: mobile genes and introns and highly variable mutation rates. Proc Natl Acad Sci USA 2000, 97:6960-6966.

10. Abdelnoor RV, Yule R, Elo A, Christensen AC, Meyer-Gausen G, Mackenzie SA: Substoichiometric shifting in the plant mitochondrial genome is influenced by a gene homologous to MutS. Proc Natl Acad Sci USA 2003, 100:5968-5973.

11. Janska H, Sarria R, Woloszynska M, Arrieta-Montiel M, Mackenzie SA: Stoichiometric shifts in the common bean mitochondrial genome leading to male sterility and spontaneous reversion to fertility. Plant Cell 1998, 10:1163-1180.

12. Schnable PS, Wise RP: The molecular basis of cytoplasmic male sterility and fertility restoration. Trends Plant Sci 1998, 3:175-180.

13. Cui $P$, Liu HT, Lin Q, Ding F, Zhuo GY, Hu SN, Liu DC, Yang WL, Zhan KH, Zhang AM, Yu J: A complete mitochondrial genome of wheat (Triticum aestivum cv. Chinese Yumai), and fast evolving mitochondrial genes in higher plants. Journal of Genetics 2009, 88:299-307.

14. Andre $C$, Levy $A$, Walbot $V$ : Small repeated sequences and the structure of plant mitochondrial genomes. Trends Genet 1992, 8:128-132.

15. Sugiyama $Y$, Watase $Y$, Nagase M, Makita $N$, Yagura $S$, Hirai A, Sugiura $M$ The complete nucleotide sequence and multipartite organization of the tobacco mitochondrial genome: comparative analysis of mitochondrial genomes in higher plants. Mol Genet Genomics 2005, 272:603-615.

16. Palmer JD, Shields CR: Tripartite structure of the Brassica campestris mitochondrial genome. Nature 1984, 307:437-440

17. Fauron $C$, Casper M, Gao Y, Moore B: The maize mitochondrial genome: dynamic, yet functional. Trends Genet 1995, 11:228-235.

18. Klein M, Eckert-Ossenkopp U, Schmiedeberg I, Brandt P, Unseld M, Brennicke A, Schuster W: Physical mapping of the mitochondrial genome of Arabidopsis thaliana by cosmid and YAC clones. Plant J 1994 6:447-455.

19. Satoh M, Nemoto Y, Kawano S, Nagata T, Hirokawa H, Kuroiwa T: Organization of heterogeneous mitochondrial DNA molecules in mitochondrial nuclei of cultured tobacco cells. Protoplasma 1993, 175:112-120. 
20. Ogihara Y, Yamazaki Y, Murai K, Kanno A, Terachi T, Shiina T, Miyashita N, Nasuda S, Nakamura C, Mori N, Takumi S, Murata M, Futo S, Tsunewaki K: Structural dynamics of cereal mitochondrial genomes as revealed by complete nucleotide sequencing of the wheat mitochondrial genome. Nucleic Acids Research 2005, 33:6235-6250.

21. Wang Z, Zou Y, Li X, Zhang Q, Chen L, Wu H, Su D, Chen Y, Guo J, Luo D, Long Y, Zhong Y, Liu YG: Cytoplasmic male sterility of rice with boro II cytoplasm is caused by a cytotoxic peptide and is restored by two related PPR motif genes via distinct modes of mRNA silencing. Plant Cell 2006, 18:676-687.

22. Kennell JC, Pring DR: Initiation and processing of atp6, T-urf13 and orf221 transcripts from mitochondria of T-cytoplasm maize. Mol Gen Genet 1989, 216:16-24.

23. Song J, Hedgcoth C: Influence of nuclear background on transcription of a chimeric gene (orf256) and coxl in fertile and cytoplasmic male sterile wheats. Genome 1994, 37:203-209.

24. Hedgcoth C, el-Shehawi AM, Wei P, Clarkson M, Tamalis D: A chimeric open reading frame associated with cytoplasmic male sterility in alloplasmic wheat with Triticum timopheevi mitochondria is present in several Triticum and Aegilops species, barley, and rye. Curr Genet 2002, 41:357-365.

25. Heazlewood JL, Whelan J, Millar AH: The products of the mitochondrial orf25 and orfB genes are Fo components in the plant F1Fo ATP synthase. FEBS Lett 2003, 540:201-205.

26. Tang HV, Pring DR, Shaw LC, Salazar RA, Muza FR, Yan B, Schertz KF: Transcript processing internal to a mitochondrial open reading frame is correlated with fertility restoration in male-sterile sorghum. Plant J 1996, 10:123-133.

27. Tang HV, Chen W, Pring DR: Mitochondrial orf107 transcription, editing, and nucleolytic cleavage conferred by the gene Rf3 are expressed in sorghum pollen. Sex Plant Reprod 1999, 12:53-59.

28. Palmer JD: Contrasting modes and tempos of genome evolution in land plant organelles. Trends Genet 1990, 6:115-120.

29. Satoh M, Kubo T, Nishizawa S, Estiati A, Itchoda N, Mikami T: The cytoplasmic male-sterile type and normal type mitochondrial genomes of sugar beet share the same complement of genes of known function but differ in the content of expressed ORFs. Mol Genet Genomics 2004, 272:247-256

30. Tian $X$, Zheng J, Hu S, Yu J: The rice mitochondrial genomes and their variations. Plant Physiol 2006, 140:401-410

31. Handa $H$ : The complete nucleotide sequence and RNA editing content of the mitochondrial genome of rapeseed (Brassica napus L.): comparative analysis of the mitochondrial genomes of rapeseed and Arabidopsis thaliana. Nucleic Acids Res 2003, 31:5907-5916.

32. Kubo T, Nishizawa S, Sugawara A, Itchoda N, Estiati A, Mikami T: The complete nucleotide sequence of the mitochondrial genome of sugar beet (Beta vulgaris L.) reveals a novel gene for tRNA(Cys)(GCA). Nucleic Acids Res 2000, 28:2571-2576.

33. Clifton SW, Minx P, Fauron CM, Gibson M, Allen JO, Sun H, Thompson M, Barbazuk WB, Kanuganti S, Tayloe C, Meyer L, Wilson RK, Newton KJ: Sequence and comparative analysis of the maize NB mitochondrial genome. Plant Physiol 2004, 136:3486-3503.

34. Fauron C, Casper M, Gao Y, Moore B: The maize mitochondrial genome: dynamic, yet functional. Trends Genet 1995, 11:228-235.

35. Wolfe KH, Li WH, Sharp PM: Rates of nucleotide substitution vary greatly among plant mitochondrial, chloroplast, and nuclear DNAs. Proc Natl Acad Sci 1987, 84:9054-9058.

36. Kuzmin EV, Duvick DN, Newton KJ: A mitochondrial mutator system in maize. Plant Physiol 2005, 137:779-789.

37. Sandhu AP, Abdelnoor RV, Mackenzie SA: Transgenic induction of mitochondrial rearrangements for cytoplasmic male sterility in crop plants. Proc Natl Acad Sci 2007, 104:1766-1770

38. Warmke HE, Lee SL: Pollen abortion in T cytoplasmic male-sterile corn (Zea mays): a suggested mechanism. Science 1978, 200:561-563.

39. Wu H, Xu H, Liu ZL, Liu YG: Molecular basis of plant cytoplasmic male sterility and fertility restoration. Chinese Bulletin of Botany 2007, 24:399-413.

40. Korth KL, Kaspi Cl, Siedow JN, Levings CS: URF13, a maize mitochondrial pore-forming protein, is oligomeric and has a mixed orientation in Escherichia coli plasma membranes. Proc Natl Acad Sci USA 1991, 88:10865-10869.
41. L'Homme Y, Stahl RJ, Li XQ, Hameed A, Brown GG: Brassica nap cytoplasmic male sterility is associated with expression of a mtDNA region containing a chimeric gene similar to the pol CMS-associated orf224 gene. Curr Genet 1997, 31:325-335.

42. Gagliardi D, Leaver CJ: Polyadenylation accelerates the degradation of the mitochondrial mRNA associated with cytoplasmic male sterility in sunflower. EMBO J 1999, 18:3757-3766.

43. Iwabuchi M, Koizuka N, Fujimoto H, Sakai T, Imamura J: Identification and expression of the Kosena radish (Raphaus sativus cv. Kosena) homologue of the Orgura radish CMS-associated gene, orf138. Plant Mol Biol 1999, 39:183-188.

44. Hanson MR, Bentolila S: Interaction of mitochondrial and nuclear genes that affect male gametophyte development. Plant Cell 2004, 16 : S154-S169.

45. Kim DH, Kang JG, Kim BD: Isolation and characterization of the cytoplasmic male sterility associated orf456 gene of chilli pepper (Capsicum annuum L.). Plant Mol Biol 2007, 63:519-532.

46. Rhoads DM, Levings CS, Siedow JN: URF13, a ligand-gated, pore-forming receptor for T-toxin in the inner membrane of $\mathrm{cms}$-T mitochondria. Journal of Bioenergetics and Biomembranes 1995, 27:437-445.

47. Siedow JN, Rhoads DM, Ward GC, Levings CS: The relationship between the mitochondrial gene T-urfl3 and fungal pathotoxin sensitivity in maize. Biochim Biophys Acta 1995, 1271:235-240.

48. Yao YQ, Zhang GH, Liu HW, Wang JW, Liu HM: Cytomorphology and cytochemical localization of K-type and T-type cytoplasmic male sterile pollens in wheat. Scientia Agricultura Sinica 2002, 35:123-126.

49. Osoegawa K, de Jong PJ: BAC library construction. Methods Mol Biol 2004, 255:1-46.

50. Ewing B, Green P: Base-calling of automated sequencer traces using phred. II. Error probabilities. Genome Res 1998, 8:186-194.

51. Gordon D, Abajian C, Green P: Consed: a graphical tool for sequence finishing. Genome Res 1998, 8:195-202.

52. Lowe TM, Eddy SR: tRNAscan-SE: a program for improved detection of transfer RNA genes in genomic sequence. Nucleic Acids Res 1997, 25:955-964.

53. Schwartz S, Einitski L, Li M, Weirauch M, Riemer C, Smit A, Green ED, Hardison RC, Miller W: MultiPipMaker and supporting tools: alignments and analysis of multiple genomic DNA sequences. Nucleic Acids Res 2003, 31:3518-3524.

54. Schwartz S, Zhang Z, Frazer KA, Smit A, Riemer C, Bouck J, Gibbs R, Hardison R, Miller W: PipMaker: a web server for aligning two genomic DNA sequences. Genome Res 2000, 10:577-586.

doi:10.1186/1471-2164-12-163

Cite this article as: Liu et al:: Comparative analysis of mitochondrial genomes between a wheat K-type cytoplasmic male sterility (CMS) line and its maintainer line. BMC Genomics 2011 12:163.

\section{Submit your next manuscript to BioMed Central and take full advantage of:}

- Convenient online submission

- Thorough peer review

- No space constraints or color figure charges

- Immediate publication on acceptance

- Inclusion in PubMed, CAS, Scopus and Google Scholar

- Research which is freely available for redistribution

Submit your manuscript at www.biomedcentral.com/submit
C Biomed Central 